\title{
THE ELECTRO-ENCEPHALOGRAM IN ACUTE HEAD INJURIES *
}

\author{
BY \\ DENIS WILLIAMS \\ Squadron Leader, Royal Air Force, V.R. \\ From the Military Hospital (Head Injuries) \\ (ReCeIved 23RD MaY, 1941)

\section{Introduction}

IN a series of papers published ten years ago Berger reported abnormally slow waves in the electro-encephalogram (e.e.g.) of patients with diverse intracranial abnormalities. This work has already been adequately reviewed (Jasper, 1937, Walter, 1938). Since then these abnormally slow changes in electrical potential have been investigated in relation to tumours, abscesses, and other local lesions (Walter, 1936, 1937), high intracranial pressure (Williams, 1939), intoxications (Gibbs, Gibbs and Lennox, 1937) and degenerative diseases (Williams and Gibbs, 1939). Similar slow waves are associated with such general cerebral disturbances as the epilepsies (Gibbs, 1939), the psychoses (Hoagland, Cameron and Rubin, 1937) and abnormal personalities (Lindsley and Cutts, 1940).

No such specific characteristics as have been described in relation to the epilepsies have been found in the abnormal slow waves resulting from demonstrable cerebral damage. It appears, on the contrary, that most conditions detrimental to cerebral activity are associated with suppression of the normal frequencies and a concomitant increase in the slower components of the frequency spectrum of the e.e.g. (Gibbs, Williams and Gibbs, 1940). These abnormal conditions range from the genetic abnormality of unstable personalities and epileptics to cerebral destruction by tumours or infection, and include transitory changes in blood constituents or cerebral blood flow. The abnormally slow waves are therefore non-specific, but there appears to be a direct relationship between their number, size, and period and the severity of cerebral change.

During an earlier investigation of intracranial abnormalities (Williams, 1939) an attempt was made to correlate electro-encephalographic changes with different pathological states, and during the course of this work it was found that cerebral trauma produced abnormally slow waves, similar to those already mentioned. These changes seemed to be more variable in extent and degree, and to have a less constant relationship to the apparent severity of the lesion, than did similar changes in other abnormal states. Head injuries are notorious

* This work was communicated to the Neurological Section of the Royal Society of Medicine on June 19th, 1941. 
for their variable clinical course, their widespread symptoms, and diverse pathological changes, and consequently for the difficulties in prognosis to which they give rise. When a large number of cases of head injury were made available as a result of the war they were therefore used to study the relationship between the injury, its clinical results, and the consequent changes in cerebral electrical activity. With this in view e.e.gs. were recorded in each case admitted to a special head injury centre. The records were repeated during the period of recovery, the results co-ordinated with other clinical investigations, and experiments undertaken to try to modify the abnormalities found. During the period of this investigation, Jasper, Kershman and Elvidge (1940) described a similar study of sixty-four patients with head injuries resulting from civil accidents, the results of which are in many respects similar to those reached in the present investigation. The present investigation differs somewhat in material and approach, and it is based on observations upon six hundred consecutive cases. The present communication deals with the changes found in those patients in this series who were examined in the acute post-traumatic stage.

\section{Material and Methods}

Material.-Seventy-four of the six hundred consecutive cases of head injury were examined by electro-encephalography within 20 days of the injury. Records were taken as soon after the injury as possible, the shortest interval being 50 minutes. The first record was taken within 24 hours in ten, and within the next 48 hours in six, cases. Twenty-four others were examined during the first week, eighteen in the second and sixteen in the third. Fifty-eight of the seventy-four were examined within 14 days of the accident. When the first record was abnormal the e.e.g. was repeated at intervals until improvement ceased. With individual modifications a record was taken daily for the first three days after a head injury, then three were obtained on alternate days, followed by two a week for another month, and thereafter one weekly or fortnightly. In thirty-one of the patients who were repeatedly investigated in this manner, 179 records were taken. Forty-two patients of the seventy-four were found to have a normal e.e.g. initially, and subsequent clinical recovery in them was uneventful. These subjects with a normal record were usually examined only once, so that they account for most of the forty-three patients with single records. The patients were unselected, and all were members of the Army and R.A.F. whose ages ranged from 18 to 60 , forty-one being between 18 and 24, and twenty-six between 25 and 30 years of age. The injuries varied from simple concussion to severe cerebral laceration with dural penetration and cerebral herniation. Detailed clinical notes were available in all cases, and in addition a clinical examination, including memory and intelligence tests, was carried out at the time of the e.e.g. investigation.

Method of recording.- The patients lay quietly at rest with the eyes closed, on an earthed metal frame trolley in a shielded room. Records were made at various times of the day and night, but when conscious the patients were 
invariably awake. Electrodes were placed on the scalp and were connected to three independent condenser coupled amplifiers activating three ink-writing oscillographs (Grass) which recorded simultaneously. Time was marked in seconds.

Electrodes.-Three types of electrode were used. (1) For routine purposes saline pad electrodes were held in position by a rubber and whalebone cap similar to that described by Walter (1937). (2) When an open wound was present the cap could not always be used, so that small solder electrodes were sealed to the scalp with collodium simplex, contact being made with Cambridge electrode jelly. (3) In unconscious patients, or where much local destruction was present, stainless steel needle electrodes attached to fine shellac coated wire were introduced into the scalp or into the lacerated tissue. Whenever needle electrodes were used surface electrodes were placed on the scalp as close to them as possible, and it was apparent that within the limits of the recording system the type of electrode did not modify the e.e.g.

Electrode placing.-In routine investigations sixteen standard positions on the scalp were used, four on each hemisphere in a line from before backwards $3 \mathrm{~cm}$. from the midline, and a similar line of four over the temporal lobe, $3 \mathrm{~cm}$. above the pinna. These electrodes were connected to the recording system, as in Fig. 1. This bipolar method of placing is convenient in routine examination of cases, but many workers (Jasper, Kershman and Elvidge, 1940) use a monopolar system, with an indifferent electrode on the ear. Although the theoretical differences between the two systems are considerable, in practice the records obtained are usually similar. Paired electrodes record the difference in potential between two areas of the cortex, while, theoretically, a single electrode records the absolute potential changes over one area, so that the signal resulting from any one wave arising under one electrode is different in the two systems. In practice the general character of a series of records is not greatly changed, for the wave frequency, voltage relative to other areas, and the proportion of the different wave forms encountered are unchanged. The difference between these systems has already been referred to (Williams, 1938), and their application has been discussed by Kornmüller and Jansen (1939). Bipolar and monopolar leads were sometimes used simultaneously in order to determine from which of a pair of electrodes an abnormal wave form was being propagated, and because the comparison of the data from the two technical methods has an application to the differential diagnosis of intracranial lesions (Jasper, Kershman and Elvidge, 1940). When local changes were found, or where there was clinical evidence of focal cerebral damage, electrodes were placed in and around the abnormal area, their relative positions being changed to suit the immediate need. Thus, in a penetrating gunshot wound of the frontal lobe a line of four electrodes was placed from $2 \mathrm{~cm}$. in front of the hair line, at $2 \mathrm{~cm}$. intervals from before backwards over the wound, the third electrode being a needle introduced into the brain protruding from the wound (Fig. 7).

Clinical examination.-At the time of the e.e.g. the site and extent of superficial cranial damage was noted and abnormal physical signs observed. The 


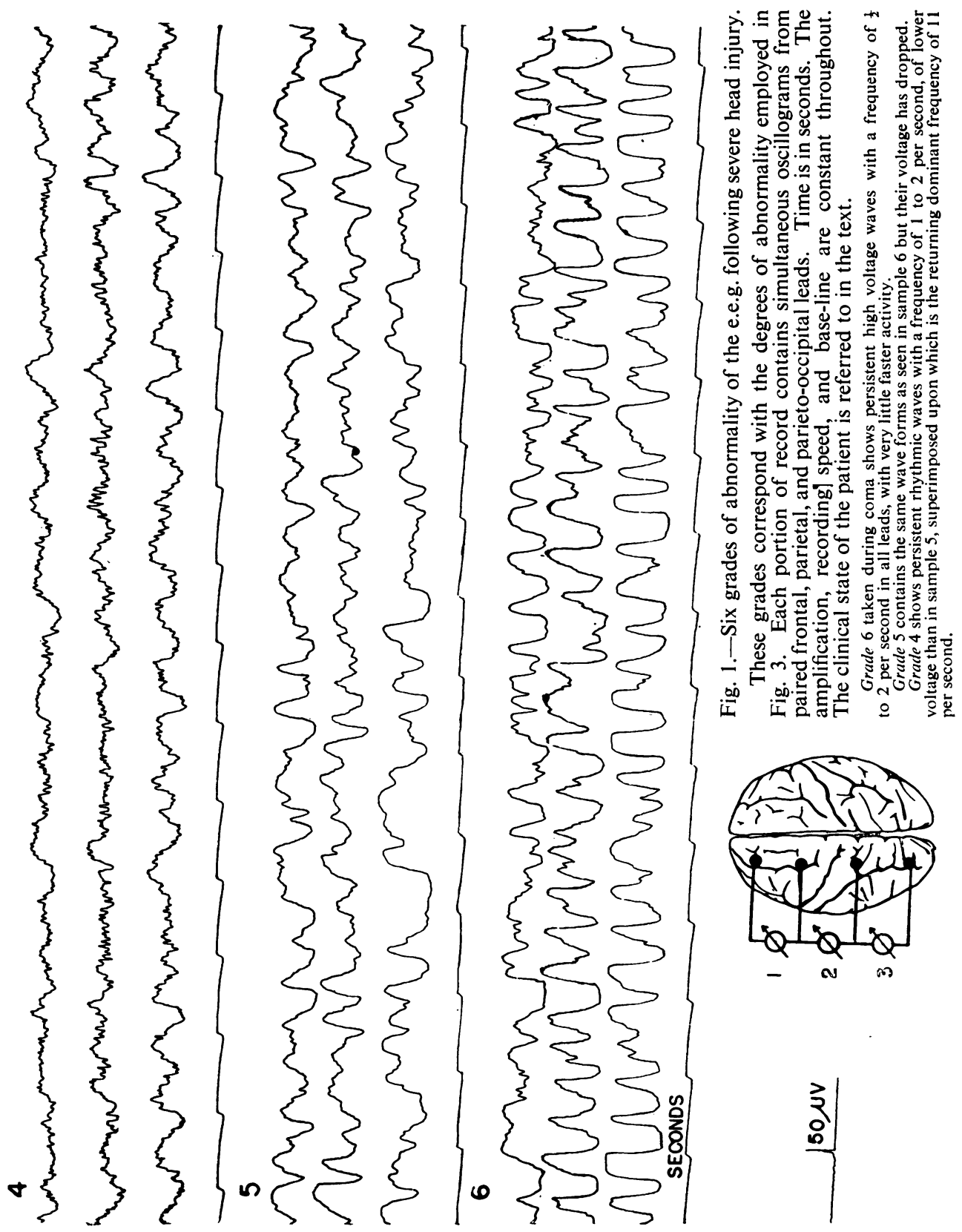


state of consciousness was assessed by a comprehensive method formulated by Group-Captain C. P. Symonds and used as a routine in the hospital. This included observation of the patient's behaviour, mood, insight, and his intellectual functions judged in the light of his past record by his orientation in time and place, awareness of current events, comprehension of complicated requests, memory, powers of calculation, and his judgment. Having obtained these data an attempt was made to correlate the following factors with changes in the e.e.g.: (a) The severity of the head injury, judged by the length of posttraumatic amnesia, and the resulting abnormalities, including cerebrospinal fluid changes. (b) Bone defects or fissure fractures. (c) Dural penetration. (d) The time after the injury. (e) Previous head injuries. ( $f$ ) The mental state of the patient at examination.

\section{Results}

Nature of the abnormal electro-encephalogram. The abnormalities seen were: (1) The appearance of widespread abnormally slow waves of cerebral electrical change, whose frequency varied from the lowest recording limits of the apparatus-less than $\frac{1}{2}$ per second-up to 7 per second (Fig. 1, samples 6 to 2). (2) A suppression of the normal dominant frequencies of $8-12$ per second (Fig. 1, samples 6 and 5). (3) Clear-cut outbursts of high voltage sine waves with a frequency of $2-3$ per second, lasting for $1-3$ seconds. These outbursts were indistinguishable from subclinical epileptic attacks. The characteristics of the abnormal waves did not differ from those described by Jasper, Kershman and Elvidge (Fig. 8).

Slow waves of the first type (Fig. 1, samples 6 to 2 ) were invariably present when there was impairment of consciousness as a result of the head injury, and their persistence, size, and period were related to the depth of unconsciousness. In cases of closed injury the disturbance was widespread throughout both hemispheres, but occasionally one or more areas of greater abnormality could be distinguished.

Coma was associated with high voltage abnormally slow waves with a period of 1 to 2 seconds and even slower frequencies below the recording limits of the apparatus, with exclusion of any fast activity. This disturbance was always generalized, and is illustrated in samples 6 and 5 in Fig. 1.

Semicoma resulting from head injury was also associated with slow waves whose frequency was higher, voltage lower and persistence less marked than those seen in coma (samples 4 and 3 in Fig. 1).

Confusion, with varying degrees of clouding of consciousness, following a head injury was accompanied by abnormally slow wave forms, which were much more variable in their incidence than those already described. Their voltage and frequency varied from second to second, but short runs of 4 to 6 sine waves, with a frequency of 2 per second, were a characteristic feature (Fig. 1, samples 4 to 2 inclusive). As confusion lightened the runs of slow waves became less evident, the frequency of the abnormal waves increased, while their voltage fell, and the unstable mixture of waves from 3 to 8 per second gradually 
disappeared. At the same time the normal frequency of 8-12 per second tended to increase in voltage and to become the dominant feature of the record (samples 2, 1 and 0 in Fig. 1).

The changes in the e.e.g. associated with an uncomplicated head injury are represented diagrammatically in Fig. 2. In this diagram an imaginary curve

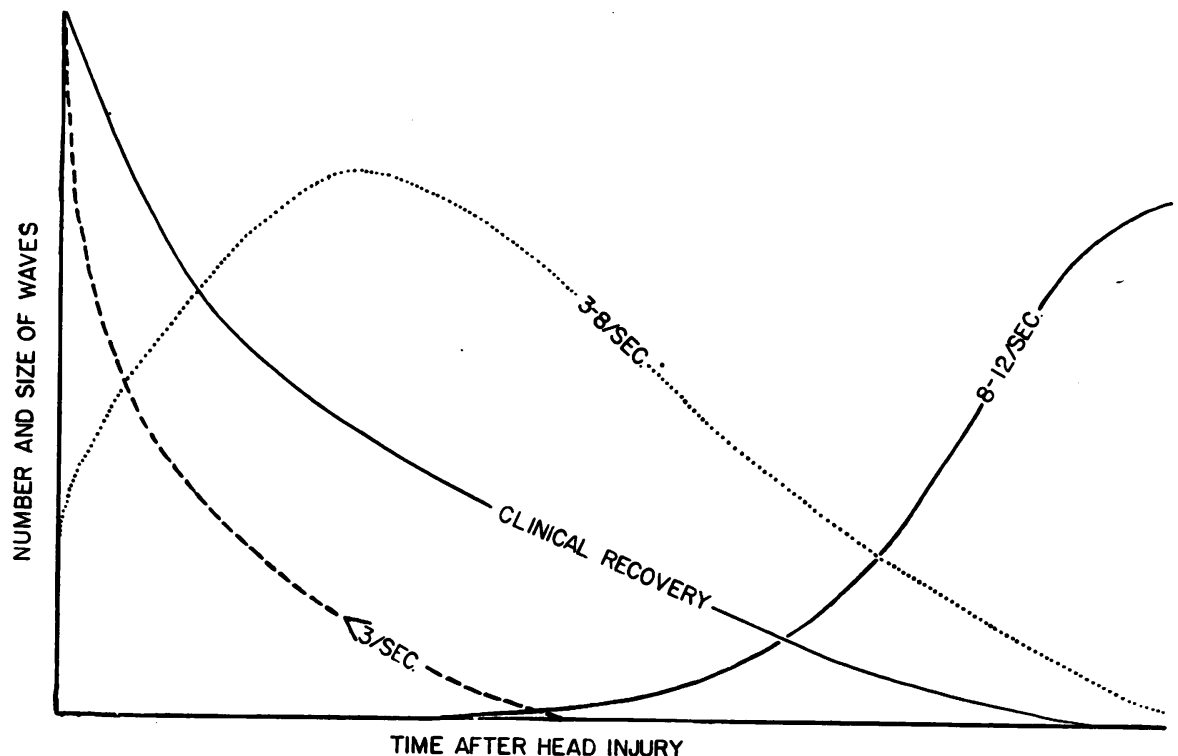

Fig. 2.-A diagrammatic representation of the usual mode of recovery of the e.e.g. in an uncomplicated head injury. A curve of clinical recovery is shown, with the curves of disappearance of the abnormal slow wave forms, and of reappearance of the normal frequencies. The increase shown in the number of medium slow waves is the result of frequency increase of the very slow waves occurring as improvement takes place. The time scale is purposely not specified, for it varies from case to case, the essential character of the curves remaining the same.

of clinical recovery is drawn. In the e.e.g. the very slow waves disappear rapidly, and as they decrease in voltage their frequency increases (Gibbs, Williams and Gibbs, 1940). This speeding up of the slower frequencies implements the number of medium-slow $3-8$ per second waves, which subside much more gradually. As the very slow waves are disappearing the normal frequencies gradually begin to return, probably in proportion to their degree of dominance in the pre-traumatic e.e.g. The disappearance of the mediumslow waves lags behind the clinical recovery in many cases, and the superimposition of the dominant 8-12 per second "alpha" rhythm upon them gives rise to the "disorganization of the alpha rhythm " referred to by Jasper, Kershman and Elvidge (1940).

Severity of head injury.-There was a positive relationship between the degree of severity of the head injury and the amount of generalized abnormality in the e.e.g. This relationship has been shown in an analysis of the total series of head injuries (Williams, 1941) and conforms to the observations of Jasper, Kershman and Elvidge (1940), and of Walter (1938), who described a similar 
relationship when investigating the effects of tumours upon the e.e.g. Of sixteen patients who had a normal e.e.g. within a few days of the injury, fifteen had a period of post-traumatic amnesia of less than 5 minutes, and the other, who had an amnesia of 10 hours, made a very rapid clinical recovery. Four patients with slight head injuries and a post-traumatic amnesia of less than 5 minutes had a normal e.e.g. within a few hours of the injury, and it remained normal until discharge from hospital. Their clinical recovery was rapid. On the other hand, some subjects with more severe head injuries maintained a grossly abnormal e.e.g. for many weeks, and thereafter the rate of recovery was so variable that it could not be predicted (Fig. 3) except by correlation with the clinical state, which it usually followed closely. The relationship between severity of injury and degree of abnormality of the e.e.g. is shown by the following brief examples:

Mild concussion (Serial Case No. 582). A corporal, aged 20, had a motor-cycle accident 18 hours before an e.e.g. was recorded. Retrograde amnesia was momentary, and post-traumatic amnesia only a few seconds. The patient was alert and normal on admission, and merely complained of local tenderness at the back of the head. The cranium was normal, no abnormal signs referable to the central nervous system were found, and consciousness was not in any way impaired. His behaviour and intellectual functions corresponded with his past record, which had been very good. He had no subsequent symptoms. No fracture was seen on X-ray, and the C.S.F. was normal in all respects. The e.e.g. recorded 18 hours after the accident was perfectly normal and is reproduced in Fig. 4 (a).

Moderately severe injury (Serial Case No. 200). An aircraftman, aged 20, had a motor-cycle accident 20 hours before an e.e.g. was recorded. There was 8 hours retrograde and 12 hours of post-traumatic amnesia. On admission he was alert and co-operative and complained of a slight headache and soreness of the right parietal region. There were no abnormal signs referable to the central nervous system, no bleeding from the ears or nose, but there was bruising of the right parietal area and right ear. The left ankle was fractured. Mentally he was normal to superficial testing. Orientation was correct in place and time, and his general information and judgment were nearly up to his estimated pre-traumatic standard. He only retained 6 digits, but performed the 100-7 test correctly in 50 seconds. An e.e.g. recorded at this time, 20 hours after the accident, is illustrated in Fig. 4 (b). There was generalized abnormality, consisting of large slow waves which occasionally appeared in outbrusts of quite high voltage, the abnormality being greatest in the right parietal region. $\mathrm{He}$ continued to have headaches for a week and could not concentrate for long. He improved steadily, and after 2 weeks his intellectual performance and power of retention had improved and he was easily able to retain material read in the newspaper and to discuss it; but he volunteered that he had not his normal grasp of more complicated ideas. He now retained 8 digits and performed the 100-7 test in 30 seconds. There were no abnormal signs at any time. Four weeks after the injury he went to a convalescent home for 10 days and from thence to sick leave and duty. The progressive improvement in the e.e.g. is represented graphically in Fig. 3.

Very severe head injury (Serial Case No. 687). A private, aged 26, had a motorcycle accident, followed by semicoma. He could be roused by shouted commands and by painful stimulation. There were no superficial injuries. He continued in semi-coma, and 3 days after the accident had 17 left-sided epileptic attacks, followed by a left hemiplegia. When first seen at this time his pulse rate was 52 , he was in coma, doubly incontinent, and could not be roused at all. There was œdema of the right parieto-occipital area. He had a flaccid left hemiplegia with absent reflexes and an extensor plantar response, a slight left facial weakness, and the left pupil was 


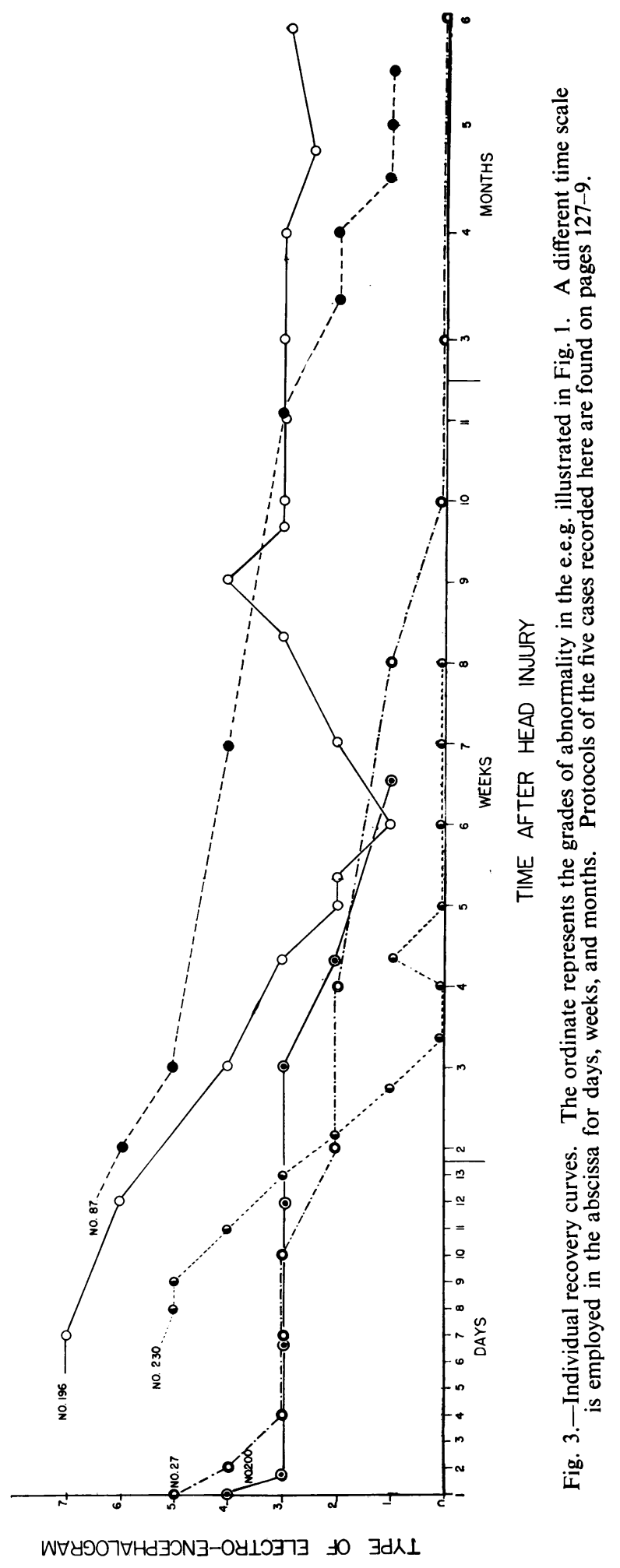


larger than the right. The C.S.F. pressure was over $300 \mathrm{~mm}$., and the fluid was yellow, but there was no block. X-ray of the skull showed multiple fissure fractures of the right parietal and occipital bones. An e.e.g. on this day showed a generalized disturbance consisting of extremely high voltage rhythmic waves with a period of one second, which persisted without interruption throughout the record. Their voltage was highest in the right frontal and parietal lobes. Improvement followed repeated drainage of C.S.F. and dehydration with mag. sulph., but the pressure remained over $300 \mathrm{~mm}$. for 7 days after his accident. He then passed from semicoma into a gradually

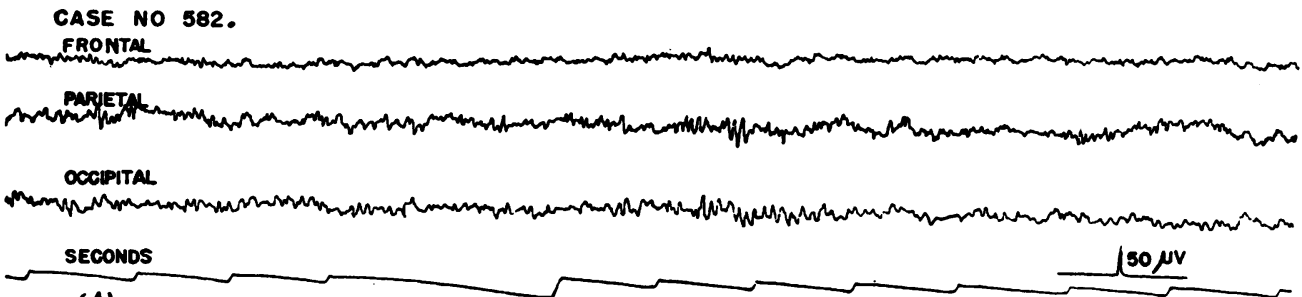

(A) MILO CONCUSSION

CASE NO 200.

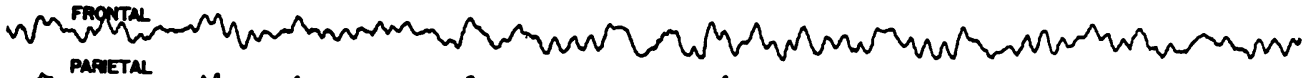

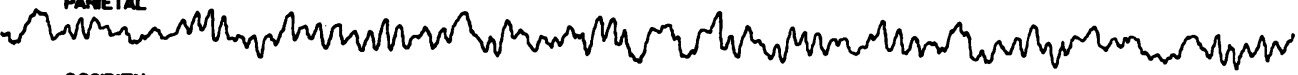

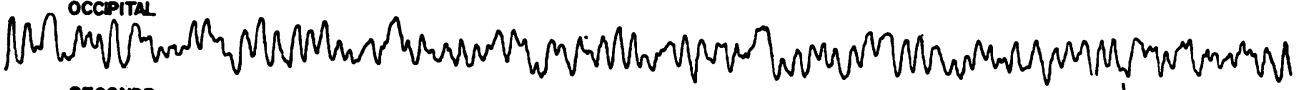
SECONDS

(B) MODERATE SEVERE HEAD INUURY

CASE NO 687.

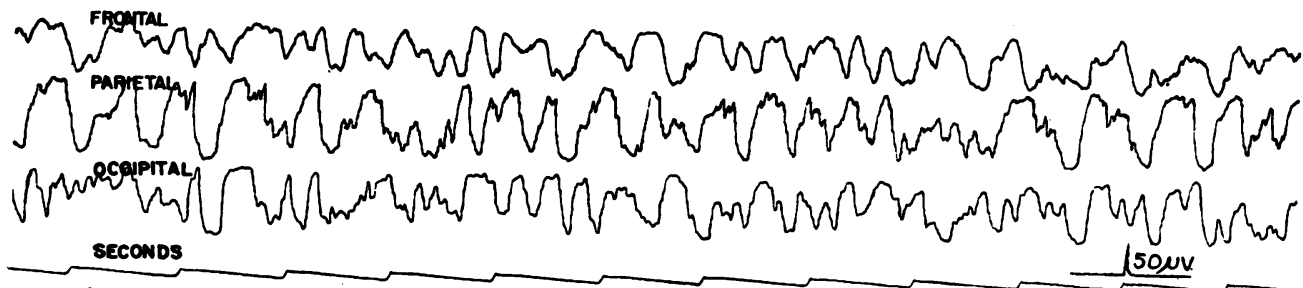

(C) VERY SEVERE HEAD INUURY

Fig. 4.-In these examples the records are taken from the same electrode placements as in Fig. 1.

(a) The e.e.g. recorded from case No. 582 (page 114), an example of mild concussion, 18 hours after the injury. The record is normal.

(b) The e.e.g. recorded from case No. 200, an instance of moderately severe head injury, 20 hours after the accident. The case history and description of the record are given on page 114 and the case is also represented in Fig. 3.

(c) The e.e.g. recorded from case No. 687, a patient with a very severe head injury, 3 days after the accident. An abstract of the case appears on page 114.

lightening state of confusion, when he was able to answer very simple questions and to perform simple commands. Three weeks after the accident he was alert and could give rational answers to questions. Retrograde amnesia was found to be over a day, and post-traumatic amnesia fourteen days. His hemiplegia steadily improved, and a left homonymous hemianopia gradually disappeared until, 4 weeks after the accident, a small homonymous defect in the left inferior quadrants was the only sequela. He was then mentally normal. A portion of the first e.e.g. is shown in Fig. 4 (c). 
Fractures.-Linear fractures of the vault or base of the skull were present in twenty-three of this series of acute head injuries. The initial abnormalities in the e.e.g. and their return to normal in these cases of fracture did not differ from the changes in other cases of equally severe head injuries.

Dural defects and open wounds.-Nine of the cases had penetrating wounds, and in two brain substance was extruding through the wound. This damage caused local changes in the e.e.g., which, as already described, was related to the severity of the cerebral destruction. Although greater abnormality was seen in the area surrounding the opening than was present elsewhere, records taken from the rest of the scalp in these recent severe head injuries always showed abnormality. When needle electrodes were introduced into the wound a focus of discharge could be observed arising from the lacerated brain (Fig. 7). As the e.e.g. and the clinical state improved (vide infra) the local abnormality which was initially obscured by the general disturbance became more and more evident until a discrete focus of abnormality could be observed in an otherwise normal series of records.

Time-interval since injury.-The generalized disturbance of cerebral rhythms occurring after head injuries gradually disappeared, and usually the e.e.g. returned to its normal condition. When an attempt was made to plot a curve of average abnormality versus time in the total series it was found that the expected recovery could not be demonstrated. This was because the persistence of the abnormality varied so greatly from case to case that the scattering of individual points was enormous. This can be seen in Fig. 3, which represents the recovery curves in five selected patients. Three of these improved rapidly, and two more slowly, while two of them were discharged before the record had returned to normal, and one even deteriorated after an initial recovery. The improvement in the e.e.g. usually followed the clinical recovery very closely, but in the late stages of recovery there was often a lag when the patient appeared normal to careful clinical testing while some residual abnormality persisted in the e.e.g. This persistent abnormality will be fully discussed in relation to chronic post-traumatic states; but it was often more spurious than real, for with the clinical methods available the psychiatrist is not able to assess the "functional reserve" of the patient's personality. This was shown by recovery, which appeared perfect in the peace of a convalescent home, giving place to obvious defects in intellectual functions and behaviour on return to the rigours of Service life. Such a response to a change in environment is compatible with the residual abnormality in the e.e.g.

Focal lesions.-The disappearance of generalized cerebral disturbances occasionally unmasked a local area of abnormality. This abnormal area coincided with an open wound when present (Figs. 5 (b), 6, and 7), but focal abnormality was also observed without localizing clinical signs (Fig. 5 (a)). The following three case reports illustrate the occurrence of abnormal discharges from a cortical region also giving abnormal physical signs, and from silent areas, without sequels in one instance and with a general intellectual defect in the other. 
Focal abnormality in a silent area without physical signs, but with corresponding personality changes (Serial Case No. 458). - A corporal, aged 50, was run down by a car. He had a laceration of the left eyebrow, with a small fracture below it. Prolonged disorientation made evaluation of retrograde amnesia difficult, but it stretched back for several weeks, and post-traumatic amnesia was for about 4 weeks. He was in semicoma at first and later showed restlessness and negativism. There were no
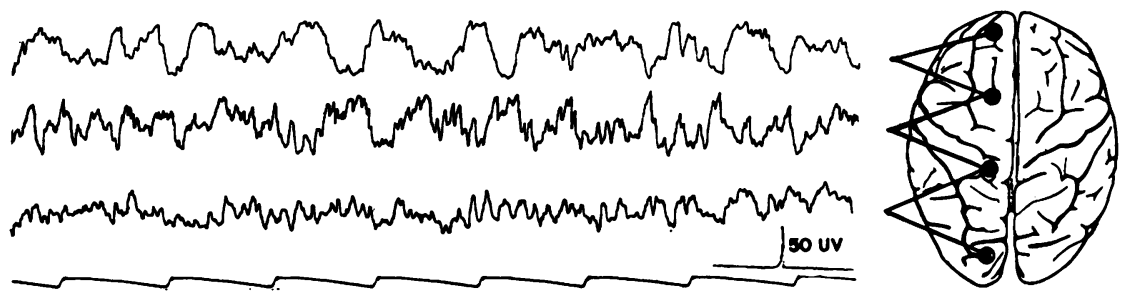

(A) SERIAL CASE NUMBER 458

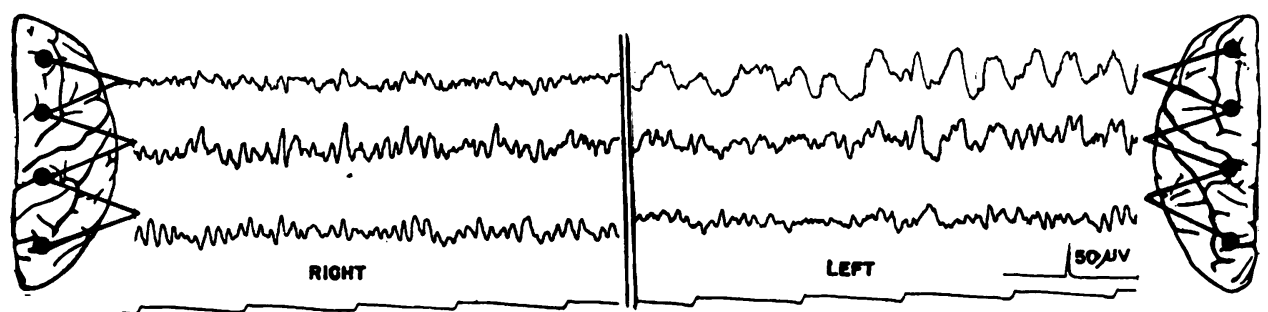

(B) SERIAL CASE NUMBER 656

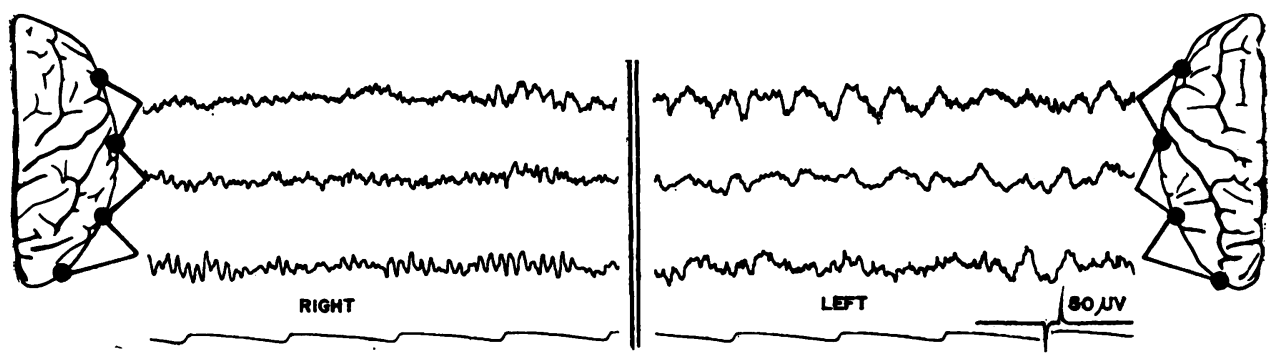

(C) SERIAL CASE NUMBer 120

Fig. 5.-Focal Cerebral Damage.

(a) Records from the left hemisphere of case No. 458 (pages 118-9). The high voltage waves with a period of one second are out of phase in the top pair of records, and are not present in the third record, indicating a focus of discharge in the left frontal lobe.

(b) Records from both hemispheres of Case No. 656 (pages 119-20). Those from the right hemisphere are normal, but that from the left frontal pole shows an abnormal rhythm of 3 per second, indicating local cerebral damage.

(c) Records from the temporal regions of both hemispheres of Case No. 120. The records from the left side are normal, but those from the right show a rhythmic 3 per second wave, indicating widespread damage in this region.

abnormal physical signs. Seen 12 days after the accident, he was drowsy, restless, uncoöperative and incontinent. He could be roused by speech, understood commands, and responded to them, but could not retain ideas for longer than a few moments. He was completely disorientated in place and time, had no insight or recall, and confabulated liberally. He would not lie still, and picked at his face and lips continuously. His past record showed him to be a physically healthy but rather 
unstable man. The C.S.F. pressure was $190 \mathrm{~mm}$., the fluid was clear, there were 3 cells and $40 \mathrm{mgm}$. of protein per $100 \mathrm{cu}$. $\mathrm{mm}$. The e.e.g. showed high voltage abnormally slow waves throughout both hemispheres. Their voltage varied between $100-200 \mu v$. and their frequency from 1 to $\frac{1}{2}$ a second. They were slower in the frontal and temporal regions than elsewhere.

A week later he was more co-operative and less confused, though still restless, incontinent, and completely disorientated. He had a little spontaneous, rational speech. The e.e.g. had improved greatly, except from the frontal lobes, which still showed much abnormality. He continued to improve slowly, and a month after the accident superficially appeared rational.

Five weeks after the accident an encephalogram showed the anterior horns of both ventricles to be dilated. The left was larger than the right, the maximal transverse diameter being $2.5 \mathrm{~cm}$. in the right and $2.8 \mathrm{~cm}$. in the left. He was quiet, docile, and
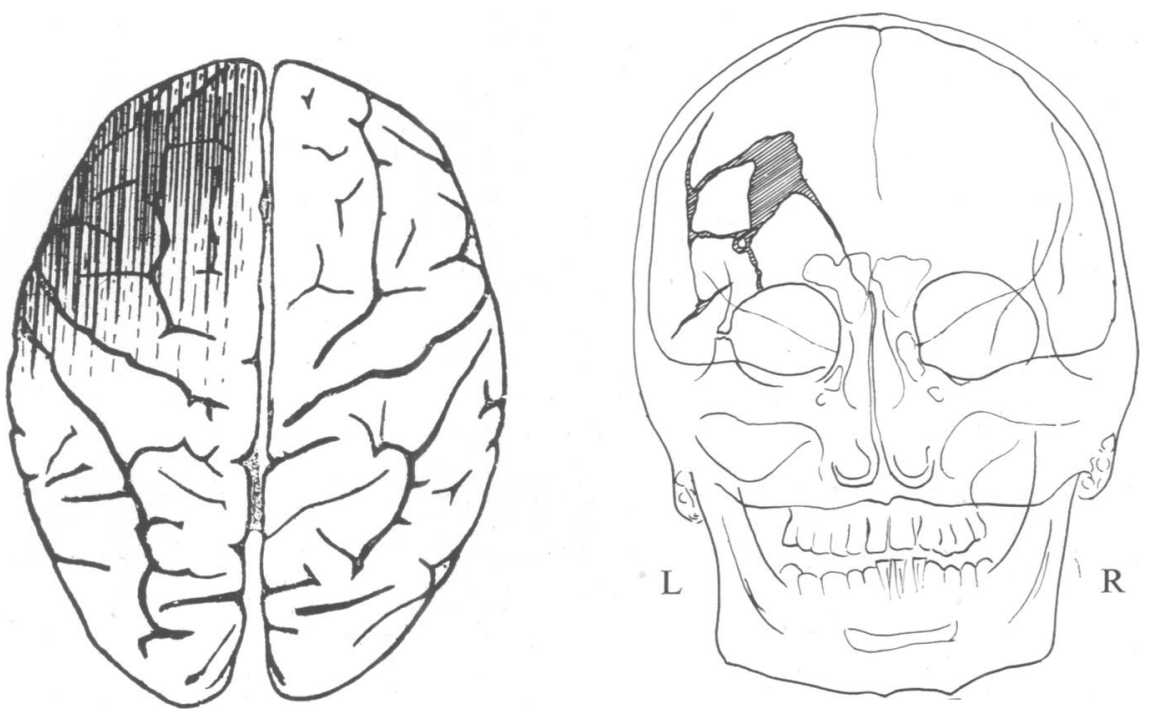

Fig. 6.-A tracing of an X-ray of the skull of Case No. 656, showing an extensive fracture of the left frontal bone, with a defect caused by operative removal of fragments, and a diagram showing the area of brain from which an abnormal e.e.g. was recorded. The situation of the abnormal shaded area corresponds closely with the position of the brain lacerated by bone fragments.

childish. The confusion was much less dense, but he was euphoric, without judgment or insight, and he had no power of recall. He could only retain 4 digits and simple calculation was impossible. Incontinence had ceased. The e.e.g. was almost normal, except from the frontal poles. Here, especially on the left side, there were medium voltage 2 to 3 per second waves (Fig. 5 (a)).

Six weeks after the accident he had improved, but was still confabulating, had no insight, and was euphoric. He could not do the simplest calculation, although recall had improved. Nine weeks after, although the e.e.g. was elsewhere normal, there was still a little residual abnormality from the left frontal lobe. He could then retain 8 digits, took an intelligent interest in international affairs, and had good judgment and insight. He was still euphoric and confabulated about the period of amnesia. Comparison of the changes in the e.e.g. recorded on 8 occasions during the period of improvement in his clinical state showed a close correlation. The clinical picture was preponderantly that of a frontal lobe syndrome, and the e.e.g. showed the abnormality to be greatest in this region.

Focal abnormality confirmed by a bone defect and exploration (Serial Case No. 656). -A private, aged 21 , was involved in a motor-cycle collision. Retrograde amnesia 
was less than 5 minutes and post-traumatic amnesia about 12 hours. He remembered everything that happened and his only complaint was soreness of the left side of his head. He had no other symptoms of any sort. He had bilateral subconjunctival hæmorrhages and bruising but no other abnormal signs, and the central nervous system was normal. X-ray showed a closed depressed fracture of the left frontal bone with a diameter of about $2 \frac{1}{2}$ in. A flap was reflected under gas and oxygen, the bone elevated, and several small pieces removed. The dura was torn and there was cerebral laceration. The dura could not be approximated, but the wound was closed in layers. Subsequently there were no symptoms or abnormal signs and the patient made an uneventful recovery. Full psychological testing 12 days after the accident showed no discrepancy between his performance and his record before the injury. An e.e.g. recorded on the same day showed considerable abnormality from an area sharply localized to the left frontal pole. The record from the right hemisphere was within normal limits, but from the left side there was a high voltage 3 per second wave in the anterior pair of electrodes, both in a row of leads near the vertex and in leads along the line of the Sylvian fissure. Fig. 5 (b) illustrates the type of abnormality

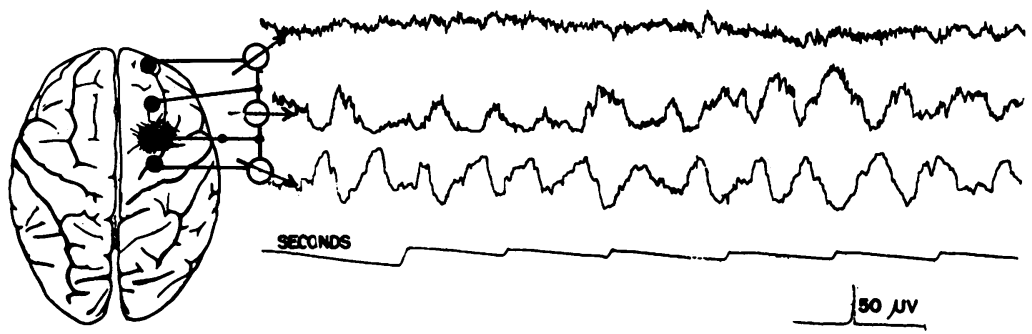

Fig. 7.-An e.e.g. obtained 12 hours after the injury, from the right frontal lobe of a patient with a penetrating gunshot wound of the head. Minute bomb fragments had entered the right frontal lobe in the shaded area, and lacerated cerebral substance was protruding through the small puncture wound. The patient was alert, mentally normal, and had no abnormal physical signs. He had no complaints of any sort. Two surface electrodes were sealed to the scalp anterior to the wound, a stainless steel electrode was placed in the lacerated brain in the wound, and a fourth electrode was sealed to the scalp in line with the others immediately behind the wound. The four electrodes were connected to the three amplifying systems in the manner shown. The record from the first pair consists of a straight base line with fast spikes caused by the activity of the frontalis muscle. The lower two records show an irregular high voltage wave, with a frequency of approximately 2 per second, which shows a persistent phase difference in the two records. This indicates an extremely localized discharge from abnormal brain under the needle electrode, the brain a short distance away being quite normal.

seen, and Fig. 6 shows the correlation between the bone defect and the area of abnormal cortex.

Focal abnormality with corresponding clinical signs (Serial Case No. 120). An aircraftman, aged 20 , was struck on the head during a bomb explosion, which he recalls. There was about 24 hours post-traumatic amnesia. He was taken to hospital in a semi-conscious state with a lacerated wound of the left parieto-occipital region. A depressed fracture was present under the laceration, and no exploration was made, the scalp wound above being cleaned and sutured. X-ray showed a depressed left parieto-occipital fracture, with a shower of bone fragments in the underlying cortex. He continued drowsy, had a flaccid right hemiplegia and a right homonymous hemianopia. Three days after the accident he had three grand mal attacks, beginning on the right side. The hemianopia began to clear up in the first week, and then the hemiplegia rapidly improved. He had no further convulsions. The C.S.F. was normal, pressure being $150 \mathrm{~mm}$. He was first seen 3 weeks after the injury. He then had intermittent headaches, and attacks of dizziness on turning quickly. There were no abnorma! signs in the central nervous system except for the slightest clumsiness of the right hand. His performance of psychological tests was poor. He retained 7 digits, but judgment, 
insight, and intelligence were not of the standard expected from his past record. Encephalography showed a normal ventricular system. He improved steadily, his intellectual level rose, and he ultimately returned to duty. The e.e.g. showed a practically normal record from the right hemisphere, but on the left side there were very well marked abnormal waves with a frequency varying from 1 to 4 per second in the occipital and posterior parietal regions. They were particularly well seen low down behind the ear. A sample of the record and a diagram of the abnormal area are shown in Fig. 5 (c).

One of the outstanding features of e.e.gs. recorded within a few hours of a head injury was the appearance of intermittent outbursts of high voltage sine waves with a frequency of 2 to 3 per second whose character was identical with that of a variant of petit mal epilepsy (Fig. 8). These outbursts usually occurred in all leads simultaneously; but occasionally they appeared in leads from different lobes independently (Fig. 8). They were found when an e.e.g. was obtained within a few hours (up to 48) after a head injury, and in one instance they lasted for 8 days. They were usually associated with an alteration in the state of consciousness of the patient, always subsided completely, and had no apparent relationship to the onset of immediate post-traumatic convulsions. Further, their presence bore no apparent relationship to the subsequent incidence of post-traumatic epilepsy as judged from the follow-up of cases (Williams, 1941). Their presence seemed to be related to the short interval elapsing between the injury and the e.e.g., for they subsided after the acute phase of the post-traumatic state had passed. This type of abnormality is in marked contrast to that seen in other cerebral abnormalities. Neoplasms cause abnormally slow waves, whose period is long in relation to their voltage (Walter, 1937), degenerative diseases are associated with persistent slow sine waves, whose outstanding characteristic is a long period (Williams and Gibbs, 1939), and high intracranial pressure is also associated with persistent slow "rollers" (Williams, 1939a). Acute cerebral abscesses are sometimes associated with bursts of relatively high voltage 2 to 3 per second waves, which emanate from the surrounding abnormal cortex, and it is probable that a tumour causing sufficiently severe damage to the surrounding brain will cause similar changes. Even so, it is only in the immediate post-traumatic period and in epilepsy that these outbursts of high voltage slow waves are so clear-cut and characteristic.

Epilepsy.-Six of the seventy-four patients with recent head injuries had grand mal epileptic fits shortly after the head injury. None had had any previous personal or family history of epilepsy, but in one case there was a strong family and personal history of migraine. Two of the six patients had mild, two moderate, and two severe, head injuries. The first fit occurred in these cases 4,7 , and 13 days and 1,2 , and 3 months after the head injury. In two of these subjects records taken between fits were characteristic of epilepsy, as subclinical epileptic outbursts were present. In two the records showed a generalized non-epileptic abnormality, and two were normal. After the changes characteristic of the first few days, no evidence of any epileptic activity was seen in the e.e.g. of any of the cases of recent head injury without fits. The two patients who showed subclinical epileptic outbursts had already had a fit 

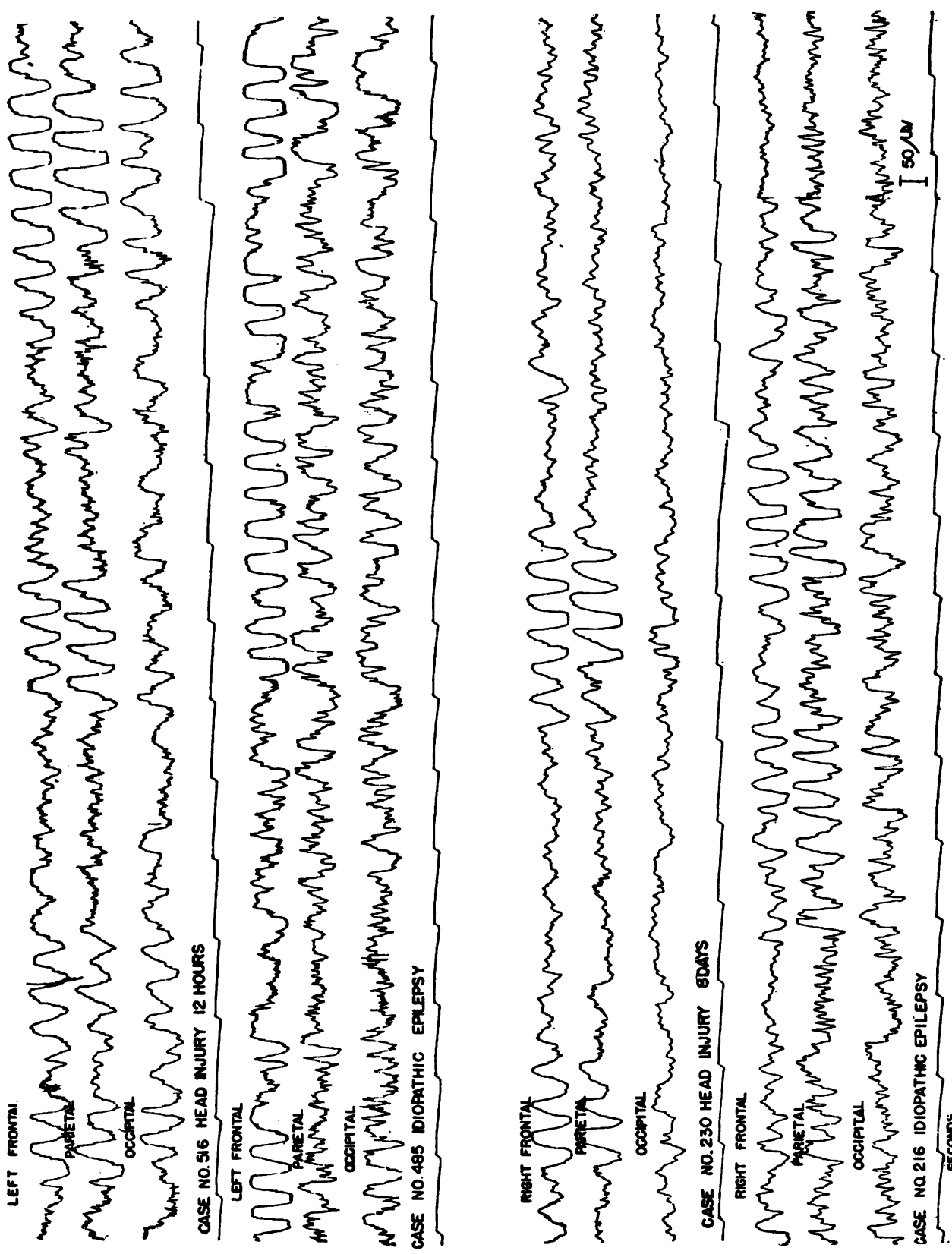

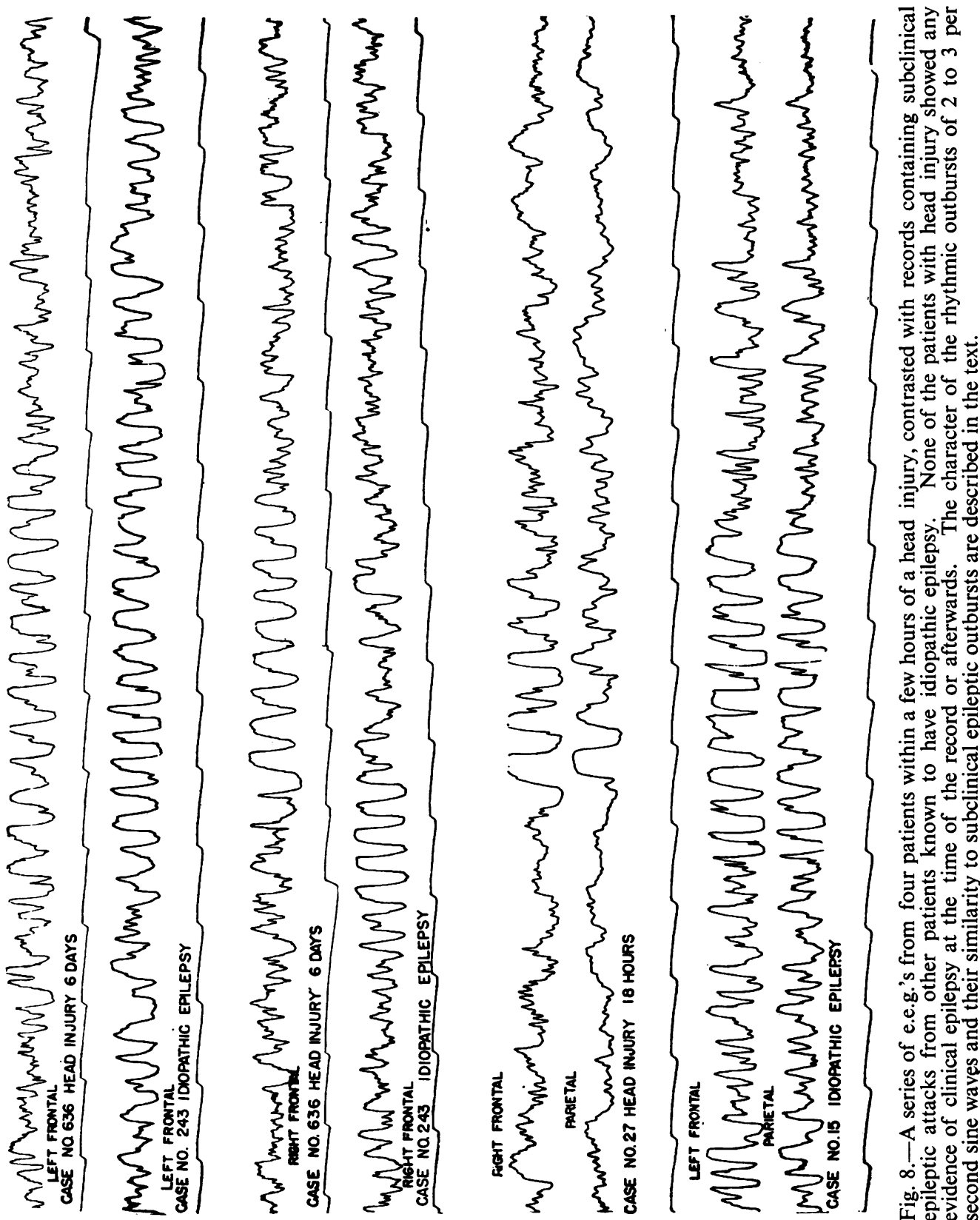

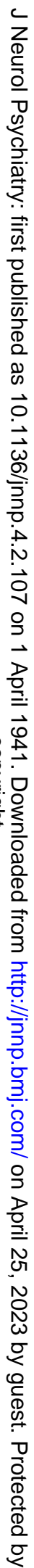


before the e.e.g. was recorded, so that it appears that the onset of post-traumatic epilepsy cannot be predicted by electro-encephalography during the acute phase of head injuries. This subject is discussed in relation to chronic posttraumatic states (Williams, 1941).

\section{Discussion}

Although the abnormal slow waves seen in the e.e.g. are non-specific, they almost invariably represent activity arising in abnormal cerebral tissue. This cerebral dysfunction may be associated either with recognizable changes in the neurones themselves, the neuroglia, the intercellular matrix, or be without any demonstrable histological abnormality. Examples of the first type are to be found around a cerebral tumour, a severe cerebral contusion, or in degenerative conditions, such as Schilder's disease. Examples of the second type are to be found among many non-deteriorated epileptics, schizophrenics, and those with abnormal personality traits. Occasionally abnormal records are obtained from apparently normal subjects. These subjects are frequently members of abnormal families and have near relatives with epilepsy, psychoses, or milder disturbances of behaviour (Lennox, Gibbs and Gibbs, 1940); but even if no such history is to be obtained, there is no prima facie reason for supposing that because they have so far been normal members of society they will continue so. It is probable that even in this group the abnormal e.e.g. demonstrates a potential abnormality which may unmask itself later in life.

It is important, therefore, to determine if an abnormal e.e.g. discovered after a head injury is the result of the injury or was present before it. In most instances, and certainly in the very acute cases, the degree of abnormality shows that the disturbance is due to the injury, and if the e.e.g. is repeated at intervals and improvement or deterioration in the record occurs, it may safely be concluded that the abnormality is a result of cerebral damage.

The results of this investigation have shown that the abnormalities in the e.e.g. correlate closely with the clinical changes resulting from the head injury, and in this they confirm the observations of Jasper, Kershman and Elvidge (1940). The e.e.g. may therefore be used to follow the progressive cerebral changes resulting from head injuries. The practical value of this is greatest when clinical evidence of damage is slight, for in the immediate post-traumatic phase of a severe head injury routine bedside observation discloses more than the e.e.g. Shortly after a slight head injury, however, the presence or absence of electro-encephalographic abnormality may be a valuable guide to the future management of the case.

As no evidence has been presented, to my knowledge, that active abnormality of cerebral tissue may persist with a normal e.e.g., a normal record after a recent head injury may be taken as indicating the absence of a progressing abnormality of cerebral tissue. Absence of abnormal waves may be associated with complete resolution or absolute destruction of damaged brain substance, for although large cerebral defects following vascular occlusion show abnormally slow waves during the period of degeneration, the e.e.g. ultimately appears 
normal, and clean surgical removal of cortex does not leave any aftermath in the e.e.g. It is evident, then, that the e.e.g., when used as an index of active cerebral abnormality after a head injury and correlated with the history and other clinical signs, will help to estimate the extent of the cerebral damage. For instance, a normal e.e.g. obtained shortly after a head injury of whatever superficial character indicates that no gross cerebral damage has occurred, while a normal record in a patient with stupor or confusion attributed to head injury throws doubt on the diagnosis of trauma, for recent damage sufficient to cause obvious impairment of consciousness is invariably associated with an abnormal e.e.g. On the other hand, an abnormal e.e.g. after an apparently trivial head injury may be due either to active cerebral change resulting from the injury or to an abnormal pre-traumatic record, the presence of which, in the light of the observations of Lennox, Gibbs and Gibbs (1940), and Lindsley and Cutt's (1940), upon the high incidence of abnormal e.e.g.'s in potentially abnormal personalities, should lead to a more guarded prognosis than might otherwise have been given. The time taken by the e.e.g. to return to normal after a head injury shows wide variations and unexpected discrepancies, but this again is fully in accordance with clinical experience, and the e.e.g. is merely another objective sign of the rate of cerebral recovery.

In view of the cellular changes which may occur after head injuries it is not surprising that the abnormalities seen in the e.e.g. are widespread. Hassin (1940) points out, from both experimental and pathological studies, that the initial changes following trauma are spread throughout the hemispheres and brain stem; and the early experiments of Scagliosi (1898) and Jacob (1912) clearly demonstrate the diffuse nature of the pathological changes. The abnormally slow waves seen in the e.e.g. almost certainly originate in cortical neurones, either as the result of damage to the cells or indirectly following interruption of projection bundles in the white matter, by section (Bishop, 1936) or by œdema and secondary changes (Williams, 1939). They may even arise as the result of involvement of the brain stem (Walter, Griffiths and Nevin, 1939), which so often occurs in head injuries (Denny-Brown, 1941).

It has been impossible so far to explain the disturbance seen in the e.e.g., in cases of head injury as the result of the direct parenchymatous degeneration (Cajal, 1928), the alteration in fibre pathways (Greenfield, 1938), the generalized œdema (Rand and Courville, 1931), the vascular changes or the petechial hæmorrhages found at necropsy. They are probably all inculpated to varying degrees in different subjects, for Hassin (1940) points out that head injuries cause widespread abnormal physico-chemical changes, whose nature is not yet understood. Trotter (1923) argued that transitory but severe cerebral anæmia might be the prime cause; Cannon (1901) related the disturbance to osmotic changes, while Dixon (1940) has recently suggested that parenchymal hypoglycæmia may result from the commotion of a head injury. Added to these are the more obvious vascular changes, cerebral disorganization by petechial hæmorrhages, and the direct physical violence upon cerebral cells as a result of mechanical agitation (Russell, 1931). The e.e.g. accurately reflects these physico-chemical changes without in any way shedding light on 
their character. As consciousness is the result of co-ordination of complex intracerebral mechanisms, the degree of its impairment indicates the general cerebral disturbance following head injury. It is therefore not surprising that changes in the state of consciousness correlate closely with changes in the e.e.g. It must be stressed in this connection that changes in physical signs other than changes in consciousness, $\mathrm{X}$-rays, and C.S.F. findings are too gross indices of damage to be of value in any but the most acute and severe stages of cerebral damage, at which time electro-encephalography is of little practical value.

The appearance of outbursts of abnormal wave forms which are indistinguishable from those found in epilepsy have been reported above, and have been commented upon by Jasper, Kershman and Elvidge (1940). They occur in the acute stage of head injuries and are not always associated with unconsciousness. A review of e.e.gs. taken from a wide range of abnormal states show that these changes are specific to severe cerebral damage and to epilepsy. The observation by Symonds (1935) of the similarity between the phenomena of concussion and the effects of an epileptic explosion is interesting in this respect. Comparing the changes in consciousness following the two conditions he pointed to a similarity between the state of automatism following mild head injuries and petit mal at one end of the scale, and between traumatic stupor and the stupor of epileptic exhaustion at the other, and he said that each grade of cerebral trauma between these extremes could be found to parallel a grade of post-epileptic disturbance. His statement that "whatever is the disturbance of function underlying traumatic unconsciousness, it is probably of the same general nature as that which results from the epileptic explosion " has been given objective support by the present observations.

Only the general disturbance resulting from head injuries has been discussed, but when physical signs are present, an area from which abnormal waves may be obtained will be found to correspond with the predicted site of damage; but local e.e.g. disturbances may be found in the frontal or occipital lobes in the absence of physical signs. A study of these local abnormalities in relation to associated psychological changes may be of considerable value in an investigation of some of the effects of head injuries.

In conclusion it is well to stress again the generalized nature of the electroencephalographic disturbances resulting from a head injury, which supports the view that cerebral concussion is the result of widespread disorganization of cerebral function and not of specific damage to single structures or isolated tissues.

\section{Summary and Conclusions}

Seventy-four patients who had received a head injury were investigated by electro-encephalography within 20 days of the injury. Ten were examined on the first day and fifty-eight within 14 days of the injury. The electro-encephalographic changes were correlated with the clinical state of the patients, judged by physical examination and psychological testing. Open and closed wounds of all degrees of severity were investigated. Widespread abnormally slow waves 
of cerebral electrical change, suppression of the normal frequencies, and clearcut outbursts of high voltage sine waves with a frequency of 2 to 3 per second indistinguishable from subclinical epileptic attacks, occurred in the acute stage.

The degree of abnormality seen in the electro-encephalogram correlated closely with the clinical state of the patient, cases of mild head injury without residual symptoms showing a normal electro-encephalogram within a few hours of the accident and severe injuries causing the abnormalities to persist for many weeks.

The rate of clinical and electro-encephalographic recovery in this series of acute head injuries corresponded closely, but there were wide individual variations in the speed of return to normal. In the later stages of recovery the electro-encephalogram sometimes remained abnormal after the patient had made a complete clinical recovery.

Although the acute changes were invariably generalized, focal areas of maximal abnormality became evident as the general disturbance subsided. When localizing clinical signs were present these corresponded with the area of abnormality seen in the electro-encephalogram. But this local abnormality was also seen in silent areas of the cerebral cortex.

The epileptic type of disturbance occurred in the immediate post-traumatic period and did not have any prognostic significance in regard to post-traumatic epilepsy.

It is concluded that the electro-encephalogram accurately reflects the abnormal cerebral state caused by a recent head injury, that the progressive changes are a direct indication of improvement or deterioration in this state, and that in the milder states of cerebral dysfunction it may demonstrate residual damage after all abnormal clinical signs have subsided. The electro-encephalogram has no value in predicting the onset of traumatic epilepsy in the acute posttraumatic period.

The electro-encephalographic apparatus was lent to the Royal Air Force by the National Hospital, Queen Square, and a grant towards the expense of this work was made by the Medical Research Council.

I am grateful to Lieut.-Colonel G. O. Chambers, M.C. for permission to publish the data included in this paper, to Group-Captain C. P. Symonds and to Colonel Hugh Cairns for extending facilities for the work, and to the Medical Officers whose cases were investigated. I am further grateful to Group-Captain C. P. Symonds and Major D. Denny-Brown for their critical interest in the work and to Miss J. K. Reynell, B.Sc. and Miss C. F. Hatfield for technical assistance.

\section{Clinical Abstracts of the Patients referred to in Fig. 3.}

Serial Case No. 27. A pilot, aged 22, was admitted 1 hour after a plane crash, with multiple wounds. Post-traumatic amnesia was 1 hour. He was drowsy but answered questions rationally, and described events up to 5 minutes before the crash. There was an extensive laceration of the forehead, which, with the other wounds, was repaired under general anæsthesia. X-rays did not show any fracture of the skull. Next day he was drowsy and a little irritable. He was correctly orientated and rational but slow in answering. The only abnormality in the central nervous system was slight weakness of the right external rectus 8 hours after the accident. 
C.S.F. pressure was $130 \mathrm{~mm}$., and the fluid was heavily bloodstained. The next day he had improved considerably, and 5 days after the accident he retained 8 digits, gave a good account of himself, and showed no intellectual defect. Subsequently he improved rapidly, and was only detained in hospital for management of a Pott's fracture. He had no symptoms or signs referable to the head injury after the first week. The e.e.g. reports are summarized below.

18 hours. Records obtained from all positions on both hemispheres were grossly abnormal. There were intermittent runs of high voltage 1 to 2 per second sine waves alternating with periods of lower voltage abnormally slow waves. There was none of the usual dominant frequency. The abnormality was most marked in the frontal lobes (Fig. 8).

2 days. The patient was much more alert and rational, and the record showed that the high voltage 1 to 2 per second waves were confined to the left frontal region; elsewhere low voltage abnormally slow waves persisted.

5 days. There was a great improvement in the clinical state and the e.e.g. showed corresponding change. The record was much more stable, the abnormally slow waves had lower voltage, were much less obvious, and there were no outbursts of high voltage 2 per second waves from the frontal lobes.

12 days. The patient was considered clinically normal. The e.e.g. had improved greatly and the only abnormality was a 5 per second wave of relatively low voltage seen most easily in the frontal lobes, especially the left.

23 days. The 5 per second waves were more intermittent and were interspersed with occasional isolated slower waves in an otherwise normal record.

50 days. The abnormal 5 per second waves and occasional 3 per second waves persisted upon an otherwise steady base line.

70 days. The e.e.g. was perfectly normal and consisted of a low voltage 11 per second frequency upon a straight base line.

This case illustrates the correlation between rapid clinical and electro-encephalographic improvement until a stage was reached when the e.e.g. lagged behind the apparent clinical improvement.

Serial Case No. 87. A private, aged 33, was run down by a car. He was admitted a week later in coma. He was subsequently found to have a retrograde amnesia of 7 days. There were multiple fractures of both frontal bones, which could be moved about en masse. He was incontinent, his pupils were dilated and sluggish to light, and there was right facial weakness, a right hemiplegia, and an extensor plantar response. The C.S.F. was clear, the pressure was $100 \mathrm{~mm}$., protein $30 \mathrm{mgm}$., and cells 7 per cu. $\mathrm{mm}$. The coma slowly lightened, but three weeks after the accident he was still deeply confused, light could not be appreciated in either eye, and the rightsided motor signs did not change. A choroidal tear and primary atrophy were found on the left eye with less marked atrophy in the right. A month after the accident he was still grossly disorientated and confused, his general physical and mental condition had not changed greatly, and he was often incontinent. The pupils were dilated and fixed to light. An encephalogram showed distortion of the right frontal pole, so burr holes were made to exclude a subdural hæmatoma. No subdural or intracerebral hæmorrhage was found, but subsequent X-rays showed a large cyst communicating with the ventricles. Two months after the accident he was still confused, disorientated, had no insight, had little spontaneous activity, and showed marked intellectual impairment. He could talk a little. A month later the mental state had improved considerably, but he was confused for recent events. $\mathrm{He}$ was euphoric, had no insight, his conversation was childish and fatuous. The right-sided signs had disappeared. Another month later intellectual improvement was obvious, he took an interest in his appearance, was less facile, and could carry on an intelligent conversation. His powers of recall were still defective. After another month he was invalided from the Army as there appeared to be permanent defects in memory, higher levels of intelligence, and personality. 
Serial Case No. 196. A sergeant, aged 24, had a motor-car accident with 6 days retrograde and 6 weeks post-traumatic amnesia. He had multiple cuts on the head and was semi-conscious and restless. He remained semi-conscious, restless, resistive, and noisy. Until admitted to this hospital 3 weeks later he was in traumatic delirium and was restless and resistive. There were no superficial wounds, but he had a slight right facial weakness with more brisk tendon jerks and an extensor plantar response on the left. The C.S.F. pressure was $170 \mathrm{~mm}$., there were $35 \mathrm{mgm}$. of protein and 3 cells per $100 \mathrm{cu}$. mm., the fluid being clear. No fracture of the skull could be demonstrated. He remained delirious, confused, and incontinent. A week later he was drowsy, confused, disorientated, and confabulating, although he could retain 8 digits. He remained confused and disorientated for 4 weeks, and during this time he confabulated and was garrulous, euphoric, and uninhibited. The left-sided pyramidal signs had disappeared. Improvement was very slow, but twelve weeks after the injury he was capable of intelligent conversation and was more reserved and stable, his intellectual functions were considered normal, and he could retain 9 digits. He was correctly orientated, his judgment was good, and he had normal insight. His past record and his family history were good, and subsequently he maintained steady progress. At no time were there any blackouts, faints or fits. The e.e.g. showed steady improvement for 6 weeks after the head injury, but then it slowly deteriorated for 3 weeks, and for the last 14 weeks of his stay in hospital it remained at a stationary level of abnormality. The significance of this cannot yet be commented upon, but the patient has so far shown no evidence of any post-traumatic epilepsy.

Serial Case No. 200. An abstract of case number 200 appears on page 116.

Serial Case No. 230. A private, aged 46, while intoxicated was knocked down by a car. Retrograde amnesia was difficult to assess, but was several days, and the post-traumatic amnesia lasted for 12 days. The patient came of a poor, unstable family, and his father and brother were epileptic. He himself was an inferior type of man, with poor intellectual development and little education. When admitted 2 days after the accident he was restless, resistive, and incontinent, he was abusive and profane, but obeyed simple commands correctly, although quite incapable of coherent conversation. There was bruising and a small laceration of the right frontal region, but the central nervous system was normal to full examination. X-ray showed a fissure fracture of the right parietal bone extending through the middle fossa into the base. An e.e.g. showed widespread abnormality, the report read: "There was a great deal of abnormality. The record showed a random mixture of abnormal waves of various frequencies and sizes, but the outstanding characteristic was the presence of outbursts of 2 per second waves of very high voltage, sometimes spreading throughout both hemispheres and sometimes localized to the frontal or occipital poles." (Fig. 8). Four days later unconsciousness was less deep, but he was still confused and disorientated in time and place. There was some dysphasia, but no abnormal signs in the central nervous system. The C.S.F. pressure was $220 \mathrm{~mm}$., the protein content was $40 \mathrm{mgm}$., and there was 1 lymphocyte per cu. $\mathrm{mm}$. Four days later he was clearer, could remember his station and duties, and the period of amnesia could be assessed. He was still dysphasic. He recovered slowly, but a month after the injury he could only retain 5 digits, his intellectual performance was very poor, and he was truculent, grumbling constantly, and had numerous neurotic traits. His past record suggested that this was his usual pre-traumatic condition, and he was invalided from the Army.

The correlation between improvement in the e.e.g. and in the clinical condition is interesting in this case. His family and past history were so bad and his previous personality and intellectual levels so low that in retrospect it appeared that he had recovered his normal pre-traumatic state much sooner than was realized. Thus the change in the e.e.g. was probably a fair reflection of the actual post-traumatic improvement, which without his past story could not be evaluated clinically. 


\section{REFERENCES}

Bishop, G. H. (1936). Cold Spring Harbor Symposia on Quantitative Biology. New York, 4, 305.

y. Cajal, S. R. (1928). Degeneration and Regeneration of the Nervous System. London, Vol. 2. Cannon, W. B. (1901). Amer. J. Physiol., 6, 91.

Denny-Brown, D. (1941). Lancet, 1, 371.

Dixon, K. C. (1940). Ibid., 2, 360.

Gibbs, F. A. (1939). J. Pediat., 15, 749.

Gibbs, F. A., Gibbs, E. L., and Lennox, W. G. (1937). Arch. int. Med., 60, 154.

Gibbs, F. A., Williams, D., and Gibbs, E. E. (1940). J. Neurophysiol., 3, 49.

Greenfield, J. G. (1938). Proc. roy. Soc. Med., 32, 43.

Hassin, G. B. (1940). Injuries of the Skull, Brain, and Spinal Cord. Brock, S., London, chapter 2, 13.

Hoagland, H., Cameron, D. E., and Rubin, M. A. (1937). Amer. J. Psychiat., 94, 183.

Jacob, A. (1912). Histol. histopath. Arb., 5, 182. (Quoted by Hassin, G. B. (1940)).

Jasper, H. H. (1937). J. gen. Psychol., 17, 29.

Jasper, H. H., Kershman, J., and Elvidge, A. (1940). Arch. Neurol. Psychiat. Chicago, 44, 328.

Kornmüller, A. E., and Jansen, R. (1939). Zeit. ges. Neurol. Psychiat., 166, 287.

Lennox, W. G., Gibbs, E. A., and Gibbs, F. A. (1940). Arch. Neurol. Psychiat. Chicago, 44, 1155.

Lindsley, D. B., and Cutts, K. K. (1940). Ibid., 44, 1199.

Rand, C. W., and Courville, C. (1931). Arch. Surg., 23, 257.

Russell, W. R. (1932). Brain, 55, 549.

Scagliosi, G. (1898). Virchow's Arch. f. path. Anat. u. Physiol. u. fur klin. Med., 122, 326.

Symonds, C. P. (1935). Lancet, 1, 486.

Trotter (1923). System of Surgery. Choyce, London, Vol. 3, 464.

Walter, W. G. (1936). Lancet, 2, 305.

- (1937). Proc. roy. Soc. Med., 30, 33. (1938). J. Neurol. Psychiat, 1, 359.

Walter, W. G., Griffiths, G. M., and Nevin, S. (1939). Brit. med. J., 1, 107.

Williams, D. (1938). Lancet, 1, 635. (1939). (a) Brain, 62, 321. (b) Unpublished observations. (1941). J. Neurol Psychiat, 4.

Williams, D. and Gibbs, F. A. (1930). Arch. Neurol., Psychiat., Chicago, 41, 519. 